infectious and parasitic $(1.57,1.07$ to 2.29$)$, genitourinary $(1.46,1.04$ to 2.04$)$, circulatory $(1.07,1.01$ to 1.12$)$, and external (non-medical) $(1.17,1.00$ to 1.37$)$ causes and decreased for deaths attributed to in situ, benign and unspecified neoplasms $(0.60,0.37$ to 0.99$)$. There was no clear relation between chemical exposure group and causespecific mortality. The mortality of each group was lower than that of the general population (SMR 0.88, 0.85 to $0.90 ; 0.82,0.80$ to 0.84 respectively). 3457 cancers were reported in Porton Down veterans and 3380 in non-Porton Down veterans. While overall cancer morbidity was the same (RR $1.00,95 \%$ CI 0.95 to 1.05), Porton Down veterans had higher rates of ill-defined malignant neoplasms (1.12; 1.02 to 1.22$)$, in situ neoplasms $(1.45 ; 1.06$ to 2.00$)$ and those of uncertain or unknown behaviour (1.32; 1.01 to 1.73$)$.

Conclusions: Mortality was slightly higher in Porton Down than non-Porton Down veterans. With the lack of information on other important factors, such as smoking or service overseas, it is not possible to attribute the small excess mortality to chemical exposures at Porton Down. Overall cancer morbidity in Porton Down veterans was no different from that in non-Porton Down veterans.

\section{EQUITY IN CANCER PATIENT SURVIVAL IN FINLAND}

A Pokhrel, T Hakulinen. Finnish Cancer Registry, Helsinki, Finland

doi:10.1136/jech.2009.096719v

Objectives: To study temporal, regional and education-related differences in cancer patient survival in Finland by site and sex.

Design: Population-based relative and cause-specific cancer survival analysis using the complete and period methods.

Setting: Five Cancer Control Regions (CCRs) comprising each of approximately one million population covering whole of Finland. Patients' level of education was studied using three classes: basic, secondary and higher obtained from population census made before diagnosis of cancer.

Participants: For regional survival, the patients were of those diagnosed with cancer in one of 14 most common primary sites in Finland in 1993-2005 and followed-up to the end of 2006. For education-related survival analyses, the patients diagnosed with cancer in 1971-2005 and vital status followed-up to the end of 2005 were considered.

Main outcome measure: The age-standardised relative (ASR) and cause-specific (ASC) survival estimates; the relative excess risk (RER) and cause-specific excess risk (CER) of death due to patients' cancer. Results: There were no significant differences in the RERs between the five CCRs except for patients with cancers of the pancreas, and patients with non-localised cancers of the breast, corpus uteri and prostate. The differences observed in 1998-2001 period window for ovarian cancer patients had disappeared in 2003-2006. The higher and secondary level educated patients had much lower CER compared to those with the basic education except for leukaemia. Women showed lower CER compared to men in each cancer sites except for cancer of urinary bladder. In 1996-2005, the differences in 5-year ASC by education level among 19 cancer sites ranged from 3 to 20 percentage points between the higher and basic level for men and from 1 to 14 percent points for women. Between the secondary and basic level, this difference ranged from 1 to 13 percentage points for men and 1 to 8 percentage points for women. A similar pattern was observed also for patients diagnosed in 1971-1985 and 1986-1995.

Conclusions: There were practically no differences in cancer survival in Finland by Cancer Control Region except for few cancer sites, indicating a uniform performance by region. However, the CER and 5 -year ASC showed a significant gradient between the highest and lowest levels of education. Women had a higher survival than the men. Despite the uniform geographical performance, there may be room for improvement in patient survival in Finland.
Friday 11 September

\section{Parallel session C}

\section{Smoking}

\section{CAN NATIONAL SMOKING PREVALENCE BE MONITORED USING PRIMARY CARE MEDICAL RECORDS DATA?}

${ }^{1} \mathrm{~L}$ Szatkowski, ${ }^{1} \mathrm{~T}$ Coleman, ${ }^{2} \mathrm{~S}$ Lewis, ${ }^{2} \mathrm{~A}$ McNeill. ${ }^{2}$ Division of Primary Care, University of Nottingham, Nottingham, UK; '2Division of Epidemiology and Public Health, University of Nottingham, Nottingham, UK

doi:10.1136/jech.2009.096719w

Background: Databases of electronic primary care records are widely used for research, but not currently as a source of national statistics on lifestyle issues such as smoking. There has been little contemporary research conducted into the quality of smoking data held within primary care, particularly since the introduction of the Quality and Outcomes Framework. This research is vital to assess the potential for using these large, longitudinal databases to monitor smoking trends. Objectives: To compare smoking data recorded within The Health Improvement Network database (THIN) with the accepted "gold standard" for measuring smoking prevalence, to investigate the potential of using THIN data to track changes in smoking prevalence. Methods: For 2000 to 2006, the annual prevalence of current, ex and never-smoking in THIN was determined, taking patients' most recent smoking-related Read codes for that year as indicative of their smoking status. These figures were compared with the expected prevalence calculated using indirect standardisation based on age, sex and country-specific smoking rates from the corresponding General Household Survey (GHS).

Results: There was generally good agreement between recording of current smoking in THIN and the expected prevalence as predicted using GHS smoking rates. For example, in 2006 the GHS-predicted prevalence of current smoking in the THIN population was $23.4 \%$ for men (women $20.7 \%$ ), with $22.6 \%$ of men (19.8\% women) actually being recorded as current smokers in their medical records. The recording of ex and never-smoking within THIN was less complete-for men the recorded prevalence of both ex and never smoking was approximately 10 percentage points lower than would be expected using GHS rates, and for women 5 percentage points lower. $17.4 \%$ of men and $8.0 \%$ of women in THIN in 2006 had no smoking status recorded in their electronic medical records.

Conclusions: These results suggest that primary care medical records within THIN can be used to identify current smokers possibly with enough accuracy for use in monitoring smoking prevalence nationally. However, recording of ex and never-smokers is less complete.

\section{THE IMPACT OF IMPLEMENTATION OF SMOKE-FREE LEGISLATION IN ENGLAND ON COTININE LEVELS IN ADULTS}

${ }^{1} \mathrm{H}$ Wardle, ' $\mathrm{S}$ Nicholson, ${ }^{2} \mathrm{~J}$ Mindell, ${ }^{1} \mathrm{R}$ Craig. ${ }^{\top}$ National Centre for Social Research (NatCen), London, UK; ${ }^{2}$ Department of Epidemiology \& Public Health, University College London, London, UK

\section{doi:10.1136/jech.2009.096719x}

Objective: To investigate the impact of the implementation on 1st July 2007 of smokefree legislation in England on tobacco smoke exposure and cotinine levels in non-smoking adults.

Design: Cross-sectional survey.

Setting: Private households in England.

Participants: Nationally-representative sample of 5330 (2585 male) self-reported non-smokers (never or ex-smokers) aged 16+ interviewed in the 2007 Health Survey for England; 3183 cotininevalidated non-smokers aged $16+(1441$ men) with a saliva sample. 